\title{
Inter-Organelle NAD Metabolism Underpinning Light Responsive NADP Dynamics in Plants
}

\author{
Shin-nosuke Hashida ${ }^{1 *}$ and Maki Kawai-Yamada ${ }^{2}$ \\ ${ }^{1}$ Environmental Science Research Laboratory, Central Research Institute of Electric Power Industry, Abiko, Japan, ${ }^{2}$ Graduate \\ School of Science and Engineering, Saitama University, Saitama, Japan
}

Upon illumination, photosystem I in chloroplasts catalyzes light-driven electron transport from plastocyanin to ferredoxin, followed by the reduction of $\mathrm{NADP}^{+}$to $\mathrm{NADPH}$ by ferredoxin:NADP ${ }^{+}$reductase for $\mathrm{CO}_{2}$ fixation. At the beginning of photosynthesis, $\mathrm{NADP}^{+}$ supply control is dominated by de novo $\mathrm{NADP}^{+}$synthesis rather than being recycled from the Calvin cycle. Importantly, ferredoxin distributes electrons to $\mathrm{NADP}^{+}$as well as to thioredoxins for light-dependent regulatory mechanisms, to cyclic electron flow for more adenosine triphosphate (ATP) production, and to several metabolites for reductive reactions. We previously demonstrated that the $\mathrm{NADP}^{+}$synthesis activity and the amount of the NADP pool size, namely the sum of $\mathrm{NADP}^{+}$and $\mathrm{NADPH}$, varies depending on the

Edited by: Paula Mulo, University of Turku, Finland

Reviewed by: Lauri Emil Nikkanen, University of Turku, Finland

Yoko Kimata-Ariga, Yamaguchi University, Japan

*Correspondence:

Shin-nosuke Hashida shashida@criepi.denken.or.jp

Specialty section: This article was submitted to Plant Physiology, a section of the journal Frontiers in Plant Science

Received: 17 May 2019 Accepted: 09 July 2019

Published: 26 July 2019

Citation:

Hashida S and Kawai-Yamada M (2019) Inter-Organelle NAD Metabolism Underpinning Light Responsive NADP Dynamics in Plants.

Front. Plant Sci. 10:960. doi: 10.3389/fp/s.2019.00960 light conditions and the ferredoxin-thioredoxin system. In addition, the regulatory mechanism of cytoplasmic $\mathrm{NAD}^{+}$supply is also involved in the chloroplastic $\mathrm{NADP}^{+}$supply control because $\mathrm{NAD}^{+}$is an essential precursor for $\mathrm{NADP}^{+}$synthesis. In this mini-review, we summarize the most recent advances on our understanding of the regulatory mechanisms of $\mathrm{NADP}^{+}$production, focusing on the interactions, crosstalk, and co-regulation between chloroplasts and the cytoplasm at the level of $\mathrm{NAD}^{+}$metabolism and molecular transport.

Keywords: $\mathrm{NAD}^{+}$supply, $\mathrm{NAD}^{+}$synthesis, $\mathrm{NADP}^{+}$synthesis, light response, linear electron transfer, photosynthesis, chloroplasts

\section{INTRODUCTION}

Nicotinamide adenine dinucleotide (NAD) and its phosphorylated form (NADP) are essential electron acceptors/donors in a broad range of cellular redox processes (Pollak et al., 2007). Interestingly, these two cofactors have rather distinct biological roles. NAD is mainly used in catabolic processes to produce cellular energy as an oxidant (Geigenberger, 2003), whereas NADP is often involved in anabolic processes to produce photosynthates, fatty acids, and carbon skeletons to support plant growth as a reductant (Kramer et al., 2004; Noctor et al., 2006). The major NADPH-generating source in darkness is the oxidative pentose phosphate pathway (OPPP) coupled to central carbon metabolism in chloroplasts (Kruger and von Schaewen, 2003). Redox regulation of OPPP enzymes in chloroplasts relies on thioredoxin (Trx) and NADPH-dependent Trx reductase C (NTRC) (Perez-Ruiz et al., 2017), thereby balancing the redox status for protection against oxidative damage (Perez-Ruiz et al., 2006). 
Under sunlight, photosynthetic electron transfer chains (PETC) are the primary source of NADPH. Plants use sunlight as a primary energy source for photosynthesis in chloroplasts (Ort and Yocum, 1996). During this process, light drives electron transfer reactions by which protons are transferred from the stroma into the thylakoid lumen generating proton motive force $(p m f)$ that is used for ATP synthesis (Kanazawa et al., 2017). Most pmf appears to be generated though linear electron flow (LEF) in which electrons released from water in photosystem II (PSII) are eventually transferred to $\mathrm{NADP}^{+}$through photosystem I (PSI; Figure 1, red line; Joliot and Joliot, 2005). Thus, photosynthesis provides NADPH as reducing power for the Calvin-Benson cycle (CBC) to assimilate carbon dioxide. After use of the reducing power in $\mathrm{CBC}, \mathrm{NADP}^{+}$is again recycled back as an electron acceptor in PSI. However, under stress conditions that weaken $\mathrm{CBC}$ enzymatic activity, a declining rate of NADPH usage and NADP recycling occurs and LEF can be overloaded resulting in generation of reactive oxygen species from the photosystems (Hajiboland, 2014; Foyer, 2018). Hence, a number of studies have elucidated the protective mechanisms, including antioxidant production, antenna size regulation, and alternative electron flow, as regulatory mechanisms of photosynthesis under natural environments (Demmig-Adams and Adams, 1992; Takahashi and Badger, 2011; Pinnola and Bassi, 2018). For example, the "malate valve" is a representative redox shuttle system of the balancing redox state in chloroplasts and exclusively exports the excess reducing power of NADPH in chloroplasts to $\mathrm{NAD}^{+}$in cytosol (Selinski and Scheibe, 2019), by which $\mathrm{NADP}^{+}$could be replenished to PSI instead of CBC.
Thus, $\mathrm{NADP}^{+}$re-supply is crucial for the redox balancing system in chloroplasts. Here, a simple question arises: why is $\mathrm{NADP}^{+}$ additionally provided for LEF via de novo $\mathrm{NADP}^{+}$synthesis in chloroplasts when or before it starves? To answer this, we need to understand the uncharacterized regulatory mechanisms of $\mathrm{NADP}^{+}$synthesis in chloroplasts.

$\mathrm{NAD}^{+}$is the only promising candidate to be the precursor for $\mathrm{NADP}^{+}$synthesis and is converted to $\mathrm{NADP}^{+}$by ATP-dependent NAD kinase (NADK) (McGuinness and Butler, 1985). Although NAD is exclusively produced in the cytosol (Hashida et al., 2009), NADP production is executed at on demand sites by various isoforms of NADK. For example in Arabidopsis, NADK1 is located in the cytosol, whereas NADK2 and NADK3 are targeted to chloroplasts and peroxisomes, respectively (Waller et al., 2009). Moreover, NADK1 and NADK2 use $\mathrm{NAD}^{+}$as the preferred substrate, whereas NADK3 demonstrates a strong preference for $\mathrm{NADH}$ (Berrin et al., 2005; Chai et al., 2005, 2006). For decades, the $\mathrm{Ca}^{2+} /$ Calmodulin (CaM)-dependence of NADK activity has been recognized (Muto and Miyachi, 1977; Anderson et al., 1980; Karita et al., 2004) and NADK2 was reported capable of binding to CaM in vitro (Turner et al., 2004). However, no candidate CaM and no response to $\mathrm{Ca}^{2+}$ in NADK2 activity have been reported elsewhere (Dell'Aglio et al., 2016). Instead of NADK2, it was recently reported that Arabidopsis P-loop ATPase has CaM-dependent NADK activity (Dell'Aglio et al., 2019). Rather than $\mathrm{Ca}^{2+}$, the light signal appears to control chloroplastic NADK2 activity and $\mathrm{NADP}^{+}$production according to the current knowledge of NADP response to light conditions (Thormahlen et al., 2017).

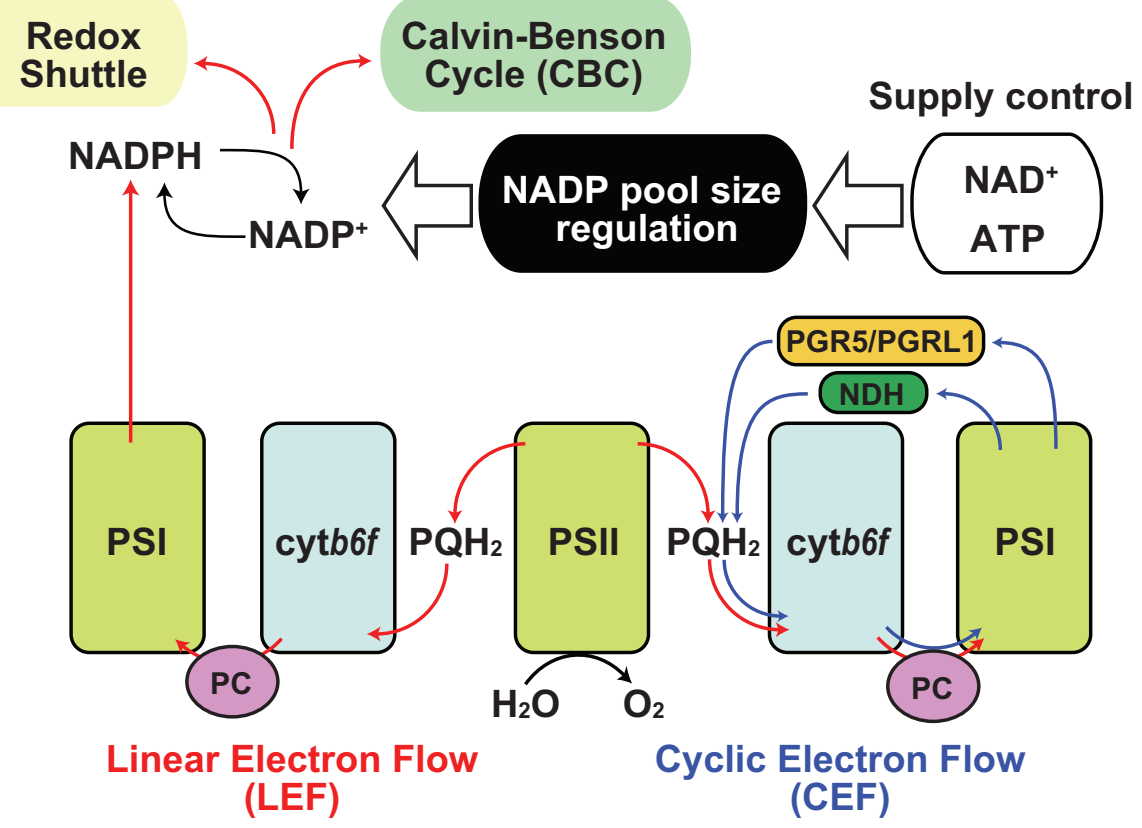

FIGURE 1 | Regulation of NADP pool size in photosynthesis. Schematic representation of the relationship between NADP pool size regulation and photosynthetic electron flow was shown. Red arrow indicates linear electron flow (LEF), and blue arrow indicates cyclic electron flow. Black arrow indicates molecular conversion. PSI, photosystem I; PSII, photosystem II; cytb ${ }_{6} f$, cytochrome $b_{6} f$ complex; PQH ${ }_{2}$, plastoquinol; PC, plastocyanin; PGR5, proton gradient regulation 5; PGRL1, PGR5-like 1; NDH, chloroplast NAD(P)H dehydrogenase-like complex. 
The goal of this mini-review is to highlight the importance of regulating $\mathrm{NAD}^{+}$supply for chloroplastic $\mathrm{NADP}^{+}$synthesis that is specific to photosynthetic organisms.

\section{DYNAMICS OF NADP POOL SIZE IN RESPONSE TO LIGHT CONDITIONS}

According to the rationale of photosynthesis, light drives $\mathrm{NADP}^{+}$ reduction, meaning NADPH generation. Most typical illustrations concerning photosynthesis demonstrate the qualitative interpretation of the photochemical process but do not give any quantitative interpretations about NADP (Figure 1). Under steady-state exposure conditions, the balance between the generation and utilization of reducing power is equilibrated by complicated interactions between LEF, CBC, redox shuttle, and cyclic electron flow (CEF), which exclusively generate pmf contributing to ATP production (Munekage et al., 2004; Alric and Johnson, 2017), such that the NADPH/NADP ${ }^{+}$ratio can remain stable. In other words, generated NADPH via PETC is immediately consumed by $\mathrm{CBC}$ and/or redox shuttle and recycled back to the photosystem as $\mathrm{NADP}^{+}$. However, photosynthesis does not occur in darkness in nature, and constant fluctuation of light intensity occurs during the day because of changes in radiation angles, cloud cover, and shade by leaf overlap in natural environments. Here, we speculate that the redox state of the NADP pool might vary in response to fluctuating light conditions and that the NADPH/NADP ${ }^{+}$ratio might be at its peak because of massive NADPH generation at very high-light intensities.
Similarly, we might misbelieve that $\mathrm{NADP}^{+}$exclusively accumulates in the chloroplasts at night because there is no light that drives PETC to generate NADPH. However, in addition to NADPH, $\mathrm{NADP}^{+}$also displays basal levels after dark acclimation for at least 30-60 min (Thormahlen et al., 2017; Hashida et al., 2018). In darkness, the redox status of NADP would be balanced by OPPP enzyme activity mediated via NTRC-dependent redox regulation (Michalska et al., 2009). At the timing of re-exposure to light, $\mathrm{NADP}^{+}$increases before NADPH because $\mathrm{NADP}^{+}$is indispensable as an electron acceptor for LEF execution. As a result, the sum of $\mathrm{NADP}^{+}$and $\mathrm{NADPH}$, here designated as the NADP pool, is dynamically adjusted to fluctuating light conditions under natural environments. These dynamics shed light on the importance of chloroplastic NADP homeostasis, including NADP pool size regulation, as well as redox regulation, in the research field of photosynthesis. Undoubtedly, the regulatory mechanism of chloroplastic NADP pool size depends on the balance between decreased and increased flow, rather than the redox interconversion between $\mathrm{NADP}^{+}$and NADPH (Figure 2).

\section{METABOLIC PATHWAYS CONTRIBUTING TO A DECREASE IN NADP POOL SIZE}

NADP metabolism, but not redox interconversion, contributes to a decrease in the NADP pool size. For example, NADP ${ }^{+}$ dephosphorylation results in $\mathrm{NAD}^{+}$generation that can be supplied for the re-synthesis of $\mathrm{NADP}^{+}$and is possibly a convenient strategy for the temporal sequestration of electron

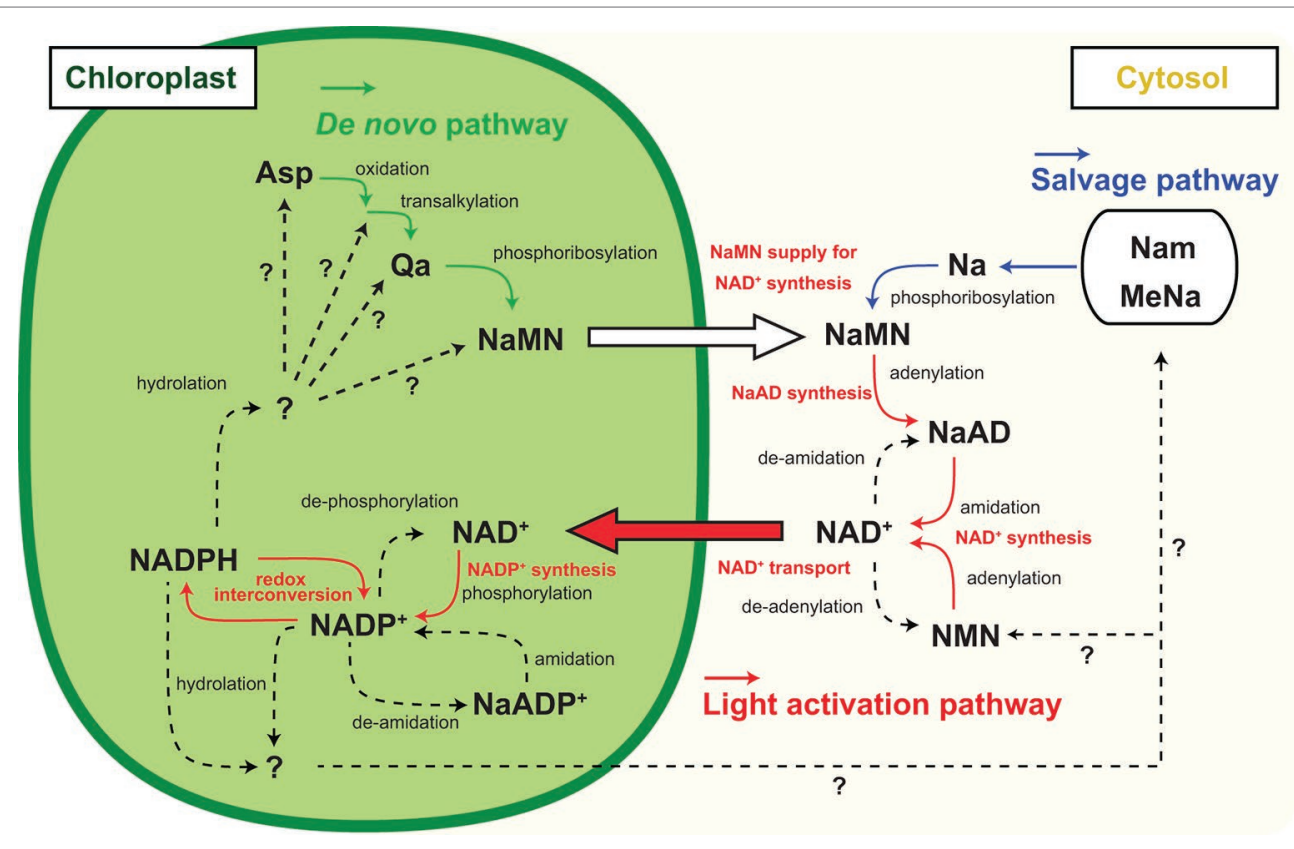

FIGURE 2 | Inter-organelle nicotinamide adenine dinucleotide (NAD) metabolism associated with chloroplastic NADP dynamics. Schematic representation of $\mathrm{NADP}^{+}$biosynthesis and NAD+ biosynthesis and part of their metabolism and inter-organelle transport. Solid arrow indicates the identified molecular conversion, and dashed arrow indicates unidentified molecular conversion. Green line highlights the de novo pathway, and blue line highlights the salvage pathway of NAD+ biosynthesis. Red line highlights the light activation pathway. Abbreviations are as shown in the text except for Nam, MeNa, and NMN indicating nicotinamide, methyl nicotinate, and nicotinamide mononucleotide, respectively. 
acceptors from photochemistry. Some research groups have reported $\mathrm{NADP}^{+}$dephosphorylation activity in plants (Gallais et al., 2000; Hunt and Gray, 2009), but no $\mathrm{NADP}^{+}$ dephosphorylation activity has been shown at present in isolated chloroplasts. Deamidation converts NADP to deamideNADP, or the so-called nicotinate adenine dinucleotide phosphate (NaADP) (Chini and Dousa, 1995). At the final step of NAD synthesis, nicotinate adenine dinucleotide (NaAD) serves in the deamidation reaction by ATP-dependent NAD synthetase (Hashida et al., 2009, 2016) so that NaADP might serve as an alternative precursor for NADP synthesis. However, NaADP molecules have never been reported in chloroplasts, and neither has NADP deamidation activity nor NaADP amidation activity been reported in chloroplasts or total leaf extracts. Nonetheless, these temporal NADP decreasing flows are attractive because they enable the re-supply of NADP for photochemistry in 1-step enzymatic reactions. On the contrary, it is known that nucleotide diphosphate linked to $\mathrm{X}$ hydrolase (NUDX) plays a significant role in NADP decrease because it cleaves the pyrophosphate bridge in the NAD skeleton (Yoshimura and Shigeoka, 2014). In Arabidopsis, AtNUDX19 aggressively participates in regulating the NADPH level as an alternative to dissipating excess reducing equivalents under intense light conditions (Maruta et al., 2016). Unlike dephosphorylation and deamidation of NADP, NUDX definitely consumes the NADP molecule, meaning that the $\mathrm{NAD}^{+}$supply is indispensable for $\mathrm{NADP}^{+}$re-synthesis as a photosynthetic electron acceptor in chloroplasts. Therefore, under high-light conditions, $\mathrm{NADP}^{+}$has to be constitutively provided to complement and maintain the NADP pool size. The fate of NADPH consumed by NUDX under high-light conditions and decreased $\mathrm{NADP}^{+}$under dark conditions has not been fully determined (Figure 2, dashed lines).

\section{REGULATORY FACTORS FOR INCREASE IN NADP POOL SIZE}

Based on enzymatic property, $\mathrm{NADP}^{+}$synthesis requires the substrates $\mathrm{NAD}^{+}$and ATP besides NADK activity (McGuinness and Butler, 1985). If one of them is lacking, $\mathrm{NADP}^{+}$supply is arrested and quiescent $\mathrm{NADP}^{+}$synthesis spontaneously decreases the pool size when the NADP pool size is determined under dynamic equilibrium. Hence, the NADP increasing flow is strictly controlled by at least three factors in chloroplasts: (1) regulation of NADK activity, (2) ATP supply, and (3) $\mathrm{NAD}^{+}$supply.

\section{REGULATION OF NADK ACTIVITY}

Notably, light-excited electrons were mainly distributed from ferredoxin (Fd) to downstream electron acceptors in two aspects, as reducing power in metabolic reactions and as redox regulator in signal transductions. Besides the reduction of $\mathrm{NADP}^{+}$by Fd-dependent $\mathrm{NADP}^{+}$reductase in LEF (Figure 1, red lines), electrons are used as reducing power in the nitrate assimilation pathway and various metabolic reactions by Fd-dependent enzymes (Hanke and Mulo, 2013). In the redox signaling network, electrons could be handed over from Fd and NADPH to thioredoxin (Trx) as reducing equivalents for the downstream signaling cascade by $\mathrm{Fd} / \mathrm{Trx}$ reductase and NTRC (Nikkanen and Rintamaki, 2019). Otherwise, electrons are transferred back to the plastoquinone pool by CEF (Figure 1, blue lines; Alric and Johnson, 2017). Thus, starvation of $\mathrm{NADP}^{+}$as an electron acceptor results in the induction of redox regulatory flow or cyclic flow. It has recently been reported that light exposure is required for the activation of NADK2 in chloroplasts and that $\mathrm{Fd} / \mathrm{Trx}$ redox signaling is directly or indirectly involved in the regulation of NADK2 activity (Hashida et al., 2018), though the regulatory mechanisms remain to be elucidated. Unlike NADK1 in cytosol and NADK3 in the peroxisome, NADK2 in chloroplasts has a long $\mathrm{N}$-terminal extension, which is conserved throughout green plants (Viridiplantae, excluding glaucophytes and rhodophytes) (Li et al., 2014), but the extended amino acid sequence is originally unnecessary for enzymatic activity. However, it is still unknown whether the extension is involved in regulating NADK2 activity.

\section{ATP SUPPLY}

CEF operates at the maximum after dark acclimation, owing to the partial inactivation of CBC (Joliot and Johnson, 2011). As NADP $^{+}$shortage caused by lack of NADK2 activation results in accumulation of reduced $\mathrm{Fd}$, it contributes to $\mathrm{CEF}$ efficiency (Breyton et al., 2006; Okegawa et al., 2008). Then, the CEF efficiency decreases shortly after light exposure (Joliot and Joliot, 2005), implying that ATP synthesis could be provided by $\mathrm{CEF}$ at the beginning of light perception for $\mathrm{NADP}^{+}$ synthesis. Therefore, CEF operation seems to be important to $\mathrm{NADP}^{+}$supply and the onset of LEF, leading to light acclimation. In fact, impairment of the CEF pathway reduced the photosynthetic rate under fluctuating light conditions, causing a decrease in plant biomass (Yamori et al., 2016), possibly owing to the delay in light acclimation, namely a delay in LEF establishment. In brief, the photochemical electron transfer network is profoundly associated with $\mathrm{NADP}^{+}$synthesis in chloroplasts.

\section{NAD ${ }^{+}$SUPPLY}

$\mathrm{NADP}^{+}$biosynthesis in chloroplasts is activated in the presence of sufficient ATP and $\mathrm{NAD}^{+}$under light conditions. Where does $\mathrm{NAD}^{+}$come from? In response to light, the chloroplastic NADP pool size doubles within $1 \mathrm{~h}$ by consuming an equivalent amount of $\mathrm{NAD}^{+}$in chloroplasts (Hashida et al., 2018). Currently, the NAD pool size (sum of $\mathrm{NAD}^{+}$and NADH) in chloroplasts is still under estimation but the sum of cellular NAD and NADP pool could increase in response to light. According to previous reports, promotion of chloroplastic NADP synthesis by NADK2 overexpression did not significantly decrease the cellular NAD pool and in contrast, enhancement of NAD 
biosynthesis increased the cellular NADP pool (Takahashi et al., 2009; Hashida et al., 2010). Thus, the increase in NADP is accompanied by an increase in the total NAD and NADP pool sizes. The total pool increase indicates activation of $\mathrm{NAD}^{+}$ biosynthesis. This activation could be stimulated by chloroplastic $\mathrm{NADP}^{+}$synthesis accompanied by $\mathrm{NAD}^{+}$uptake into chloroplasts (Figure 2). As NAD biosynthesis exclusively occurs outside of chloroplasts (Hashida et al., 2010; Di Martino and Pallotta, 2011), the cytoplasmic NAD pool could decrease by the uptake. Therefore, the mechanism of $\mathrm{NAD}^{+}$supply underpins the light responsive NADP increase for LEF in photosynthesis, and chloroplastic $\mathrm{NAD}^{+}$transportation is a crucial pathway for light adaptation in terrestrial plants (Hashida et al., 2010, 2016; Vialet-Chabrand et al., 2017). However, chloroplasts have been less investigated with respect to $\mathrm{NAD}^{+}$transport compared to mitochondria and peroxisomes (Palmieri et al., 2008; Agrimi et al., 2012). In Arabidopsis, NDT1 encodes a carrier protein that is capable of transporting $\mathrm{NAD}^{+}$and targeting it to the inner membrane of chloroplasts (Todisco et al., 2006; Palmieri et al., 2009). At present, the physiological functions in chloroplastic $\mathrm{NAD}^{+}$transportation are largely unknown. Surprisingly, a mutant lacking chloroplastic NADK activity, nadk2, can survive under light and conditionally grow to a size comparable to wild type, albeit having a pale green leaf (Chai et al., 2005; Takahashi et al., 2009), implying a possible complement of $\mathrm{NADP}^{+}$to chloroplasts from cytosol in the nadk2 mutant. However, neither NDT1 nor NDT2 transports $\mathrm{NADP}^{+}$or NADPH according to biochemical experiments (Palmieri et al., 2009). Otherwise, $\mathrm{NAD}^{+}$in chloroplasts may act as an inefficient but valuable alternative for $\mathrm{NADP}^{+}$in photosynthetic electron transport. Because, nadk2 showed a larger NAD pool size than the wild type (Takahashi et al., 2006), light-driven chloroplastic $\mathrm{NAD}^{+}$transport could be promoted and the chloroplastic NAD pool might cover the increase.

\section{WHICH PATHWAY IS CRUCIAL FOR NAD ${ }^{+}$SUPPLY?}

$\mathrm{NAD}^{+}$also has a significant function in photosynthesis through regulation of various $\mathrm{CBC}$ enzymes; for example, via the assembly of the protein complex glyceraldehyde-3-phosphate dehydrogenase and phosphoribulokinase mediated by CP12 protein (Wedel et al., 1997). Therefore, chloroplasts contain considerable amounts of $\mathrm{NAD}^{+}$and chloroplastic $\mathrm{NAD}^{+}$ transportation is not just for $\mathrm{NADP}^{+}$synthesis. Nevertheless, the basal level of $\mathrm{NAD}^{+}$cannot be accounted for by the increased $\mathrm{NADP}^{+}$level in response to light, suggesting that $\mathrm{NAD}^{+}$biosynthesis is also required for light acclimation (Figure 2, orange lines and captions). In the final two steps of $\mathrm{NAD}^{+}$biosynthesis, nicotinate mononucleotide (NaMN) is transformed via adenylation to nicotinate adenine dinucleotide (NaAD), followed by amidation of $\mathrm{NaAD}$ to $\mathrm{NAD}^{+}$. However, enhancement of enzymatic activity in these steps does not particularly contribute to the NAD pool size (Hashida et al., 2010, 2016). Here, NaMN is of particular importance as an intermediate, which is a product of two independent pathways, the de novo and the salvage pathway. In heterologous systems, quinolinate $(\mathrm{Qa})$ application with E. coli nadC gene encoding Qa phosphoribosyl transferase (QPT) successively increases the NAD pool size (Petriacq et al., 2012). Therefore, the supply of $\mathrm{NaMN}$ is a key regulator of cellular NAD pool size. In brief, the light responsive NADP increase in chloroplasts will provoke NaMN supply for $\mathrm{NAD}^{+}$production. According to current knowledge, de novo synthesis of $\mathrm{NaMN}$ is executed in chloroplasts, and the generated NaMN is exported to cytosol and salvage synthesis of NaMN from nicotinate is conducted in the cytosol (Katoh et al., 2006). Therefore, the de novo pathway rather than the salvage pathway has better linkage to chloroplastic events (Figure 2, green and red lines).

According to a recent report, L-Asp oxidase (LASPO), which is the first enzyme of de novo $\mathrm{NAD}^{+}$biosynthesis and converts L-Asp to imino-Asp, has intriguing biochemical properties associated with the light/dark condition (Hao et al., 2018). LASPO activity may be inhibited at night and stimulated by light exposure, as well as NADK2. Moreover, $\mathrm{NADP}^{+}$can be a competitive inhibitor for LASPO, suggesting that an early step of the de novo $\mathrm{NAD}^{+}$biosynthesis is orchestrated by chloroplastic $\mathrm{NADP}^{+}$status. As the next step of de novo $\mathrm{NAD}^{+}$biosynthesis, Qa synthase (QS) produces Qa from imino-Asp. Interestingly, QS protein harbors the cys desulfurase domain that stimulates reconstitution of the oxygen-sensitive Fe-S cluster and QS as part of the cys desulfurase complex (Murthy et al., 2007; Schippers et al., 2008). Hence, QS may monitor chloroplastic redox status in a Cys desulfurase complex and a reductive environment could stimulate QS activity. In chloroplasts, a Fe-S cluster and assembly are involved in the redox regulatory network downstream of Fd in PSI photochemistry (Van Hoewyk et al., 2007). Because the $\mathrm{Fd} /$ thioredoxin- $m$ redox regulatory pathway negatively regulates $\mathrm{NADP}^{+}$synthesis (Hashida et al., 2018), these opposite regulations may coordinate the precursor supply for de novo $\mathrm{NAD}^{+}$biosynthesis that is required for $\mathrm{NADP}^{+}$synthesis to save excess energy (ATP) consumption. Further, the above-mentioned heterologous system uses cytoplasmic ATP for NaMN synthesis. Therefore, as is the case of NADK in chloroplasts, ATP supply could be a ratelimiting factor for QPT activity. Although NaMN needs to be exported to the cytoplasm from chloroplasts for the synthesis of $\mathrm{NAD}^{+}$, the transport mechanism is unknown. Here, NDT1 could be a candidate NaMN transporter in addition to the ability of $\mathrm{NAD}^{+}$transport because NDT1 protein can mediate NaMN as well (Palmieri et al., 2009). In brief, the light responsive NADP increase could be underpinned by the export of NaMN from chloroplasts to cytosol and the import of $\mathrm{NAD}^{+}$from the cytosol to chloroplasts.

\section{CONCLUSION AND PERSPECTIVES}

Although genes associated with $\mathrm{NAD}^{+}$and $\mathrm{NADP}^{+}$biosynthesis and metabolism have been continuously characterized and identified for decades, their regulators and transport 
mechanisms remain largely unknown. Because $\mathrm{NADP}^{+}$ biosynthesis is mainly supported by the amount of available $\mathrm{NAD}^{+}, \mathrm{NAD}^{+}$supply is the key regulator of $\mathrm{NADP}^{+}$synthesis. Importantly, $\mathrm{NADP}^{+}$synthesis proceeds on demand even though $\mathrm{NAD}^{+}$synthesis is exclusively carried out in the cytosol. Therefore, inter-organelle signaling is rather important to communicate $\mathrm{NAD}^{+}$demand for each location where $\mathrm{NADP}^{+}$synthesis occurs. Recent studies have revealed the existence of a dynamic response of the chloroplastic NADP pool to light, underpinning NAD and NADP metabolism including degradation, metabolism, and biosynthesis. Moreover, the early steps of de novo $\mathrm{NAD}^{+}$synthesis in chloroplasts appear to be controlled by redox regulation. Future dissection of inter-organelle NAD and NADP metabolism is thus essential for understanding the fate of chloroplastic NADP under various light conditions.

\section{REFERENCES}

Agrimi, G., Russo, A., Pierri, C. L., and Palmieri, F. (2012). The peroxisomal $\mathrm{NAD}^{+}$carrier of Arabidopsis thaliana transports coenzyme A and its derivatives. J. Bioenerg. Biomembr. 44, 333-340. doi: 10.1007/s10863-012-9445-0

Alric, J., and Johnson, X. (2017). Alternative electron transport pathways in photosynthesis: a confluence of regulation. Curr. Opin. Plant Biol. 37, 78-86. doi: 10.1016/j.pbi.2017.03.014

Anderson, J. M., Charbonneau, H., Jones, H. P., McCann, R. O., and Cormier, M. J. (1980). Characterization of the plant nicotinamide adenine dinucleotide kinase activator protein and its identification as calmodulin. Biochemistry 19, 3113-3120.

Berrin, J. G., Pierrugues, O., Brutesco, C., Alonso, B., Montillet, J. L., Roby, D., et al. (2005). Stress induces the expression of AtNADK-1, a gene encoding a $\mathrm{NAD}(\mathrm{H})$ kinase in Arabidopsis thaliana. Mol. Gen. Genomics. 273, 10-19. doi: 10.1007/s00438-005-1113-1

Breyton, C., Nandha, B., Johnson, G. N., Joliot, P., and Finazzi, G. (2006). Redox modulation of cyclic electron flow around photosystem I in C3 plants. Biochemistry 45, 13465-13475. doi: 10.1021/bi061439s

Chai, M. F., Chen, Q. J., An, R., Chen, Y. M., Chen, J., and Wang, X. C. (2005). NADK2, an Arabidopsis chloroplastic NAD kinase, plays a vital role in both chlorophyll synthesis and chloroplast protection. Plant Mol. Biol. 59, 553-564. doi: 10.1007/s11103-005-6802-y

Chai, M. F., Wei, P. C., Chen, Q. J., An, R., Chen, J., Yang, S., et al. (2006). NADK3, a novel cytoplasmic source of NADPH, is required under conditions of oxidative stress and modulates abscisic acid responses in Arabidopsis. Plant J. 47, 665-674. doi: 10.1111/j.1365-313X.2006.02816.x

Chini, E. N., and Dousa, T. P. (1995). Enzymatic synthesis and degradation of nicotinate adenine dinucleotide phosphate (NAADP), a $\mathrm{Ca}(2+)$-releasing agonist, in rat tissues. Biochem. Biophys. Res. Commun. 209, 167-174.

Dell'Aglio, E., Giustini, C., Kraut, A., Couté, Y., Mazars, C., Matringe, M., et al. (2019). Identification of the calmodulin-dependent $\mathrm{NAD}^{+}$kinase sustaining the elicitor-induced oxidative burst in plants. bioRxiv [Preprint]. doi: 10.1101/521658

Dell'Aglio, E., Salvi, D., Kraut, A., Baudet, M., Macherel, D., Neveu, M., et al. (2016). No plastidial calmodulin-like proteins detected by two targeted massspectrometry approaches and GFP fusion proteins. New Negat. Plant Sci. 3-4, 19-26. doi: 10.1016/j.neps.2016.08.001

Demmig-Adams, B., and Adams, W. W. I. (1992). Photoprotection and other responses of plants to high light stress. Annu. Rev. Plant Physiol. Plant Mol. Biol. 43, 599-626. doi: 10.1146/annurev.pp.43.060192.003123

Di Martino, C., and Pallotta, M. L. (2011). Mitochondria-localized NAD biosynthesis by nicotinamide mononucleotide adenylyltransferase in Jerusalem artichoke (Helianthus tuberosus L.) heterotrophic tissues. Planta 234, 657-670. doi: 10.1007/s00425-011-1428-6

Foyer, C. H. (2018). Reactive oxygen species, oxidative signaling and the regulation of photosynthesis. Environ. Exp. Bot. 154, 134-142. doi: 10.1016/j. envexpbot.2018.05.003

\section{AUTHOR CONTRIBUTIONS}

All authors listed have made a substantial, direct and intellectual contribution to the work, and approved it for publication.

\section{FUNDING}

This work was supported by the JSPS KAKENHI Grant Number $17 \mathrm{H} 05714$ and $19 \mathrm{H} 04715$ to MK-Y.

\section{ACKNOWLEDGMENTS}

We would like to thank Editage (www.editage.com) for English language editing.

Gallais, S., de Crescenzo, M. A., and Laval-Martin, D. L. (2000). Evidence of active $\mathrm{NADP}(+)$ phosphatase in dormant seeds of Avena sativa L. J. Exp. Bot. 51, 1389-1394. doi: 10.1093/jxb/51.349.1389

Geigenberger, P. (2003). Response of plant metabolism to too little oxygen. Curr. Opin. Plant Biol. 6, 247-256. doi: 10.1016/S1369-5266(03)00038-4

Hajiboland, R. (2014). "Chapter 1 - reactive oxygen species and photosynthesis" in Oxidative damage to plants. ed. P. Ahmad (San Diego: Academic Press), $1-63$.

Hanke, G., and Mulo, P. (2013). Plant type ferredoxins and ferredoxin-dependent metabolism. Plant Cell Environ. 36, 1071-1084. doi: 10.1111/pce.12046

Hao, J., Petriacq, P., de Bont, L., Hodges, M., and Gakiere, B. (2018). Characterization of 1-aspartate oxidase from Arabidopsis thaliana. Plant Sci. 271, 133-142. doi: 10.1016/j.plantsci.2018.03.016

Hashida, S. N., Itami, T., Takahara, K., Hirabayashi, T., Uchimiya, H., and Kawai-Yamada, M. (2016). Increased rate of NAD metabolism shortens plant longevity by accelerating developmental senescence in Arabidopsis. Plant Cell Physiol. 57, 2427-2439. doi: 10.1093/pcp/pcw155

Hashida, S. N., Itami, T., Takahashi, H., Takahara, K., Nagano, M., Kawai-Yamada, M., et al. (2010). Nicotinate/nicotinamide mononucleotide adenyltransferase-mediated regulation of NAD biosynthesis protects guard cells from reactive oxygen species in ABA-mediated stomatal movement in Arabidopsis. J. Exp. Bot. 61, 3813-3825. doi: 10.1093/jxb/erq190

Hashida, S. N., Miyagi, A., Nishiyama, M., Yoshida, K., Hisabori, T., and KawaiYamada, M. (2018). Ferredoxin/thioredoxin system plays an important role in the chloroplastic NADP status of Arabidopsis. Plant J. 95, 947-960. doi: 10.1111/tpj.14000

Hashida, S. N., Takahashi, H., and Uchimiya, H. (2009). The role of NAD biosynthesis in plant development and stress response. Ann. Bot. 103, 819-824. doi: 10.1093/aob/mcp019

Hunt, L., and Gray, J. E. (2009). The relationship between pyridine nucleotides and seed dormancy. New Phytol. 181, 62-70. doi: 10.1111/j.1469-8137.2008.02641.x

Joliot, P., and Johnson, G. N. (2011). Regulation of cyclic and linear electron flow in higher plants. Proc. Natl. Acad. Sci. USA 108, 13317-13322. doi: $10.1073 /$ pnas. 1110189108

Joliot, P., and Joliot, A. (2005). Quantification of cyclic and linear flows in plants. Proc. Natl. Acad. Sci. USA 102, 4913-4918. doi: 10.1073/pnas.0501268102

Kanazawa, A., Ostendorf, E., Kohzuma, K., Hoh, D., Strand, D. D., Sato-Cruz, M., et al. (2017). Chloroplast ATP synthase modulation of the thylakoid proton motive force: implications for photosystem I and photosystem II photoprotection. Front. Plant Sci. 8:719. doi: 10.3389/fpls.2017.00719

Karita, E., Yamakawa, H., Mitsuhara, I., Kuchitsu, K., and Ohashi, Y. (2004) Three types of tobacco calmodulins characteristically activate plant NAD kinase at different $\mathrm{Ca} 2+$ concentrations and pHs. Plant Cell Physiol. 45, 1371-1379. doi: 10.1093/pcp/pch158

Katoh, A., Uenohara, K., Akita, M., and Hashimoto, T. (2006). Early steps in the biosynthesis of NAD in Arabidopsis start with aspartate and occur in the plastid. Plant Physiol. 141, 851-857. doi: 10.1104/pp.106.081091 
Kramer, D. M., Avenson, T. J., and Edwards, G. E. (2004). Dynamic flexibility in the light reactions of photosynthesis governed by both electron and proton transfer reactions. Trends Plant Sci. 9, 349-357. doi: 10.1016/j.tplants.2004.05.001

Kruger, N. J., and von Schaewen, A. (2003). The oxidative pentose phosphate pathway: structure and organisation. Curr. Opin. Plant Biol. 6, 236-246. doi: 10.1016/S1369-5266(03)00039-6

Li, W. Y., Wang, X., Li, R., Li, W. Q., and Chen, K. M. (2014). Genome-wide analysis of the NADK gene family in plants. PLoS One 9:e101051. doi: 10.1371/journal.pone.0116565

Maruta, T., Ogawa, T., Tsujimura, M., Ikemoto, K., Yoshida, T., Takahashi, H., et al. (2016). Loss-of-function of an Arabidopsis NADPH pyrophosphohydrolase, AtNUDX19, impacts on the pyridine nucleotides status and confers photooxidative stress tolerance. Sci. Rep. 6:37432. doi: 10.1038/srep37432

McGuinness, E. T., and Butler, J. R. (1985). NAD+ kinase--a review. Int. J. Biochem. 17, 1-11. doi: 10.1016/0020-711X(85)90079-5

Michalska, J., Zauber, H., Buchanan, B. B., Cejudo, F. J., and Geigenberger, P. (2009). NTRC links built-in thioredoxin to light and sucrose in regulating starch synthesis in chloroplasts and amyloplasts. Proc. Natl. Acad. Sci. USA 106, 9908-9913. doi: 10.1073/pnas.0903559106

Munekage, Y., Hashimoto, M., Miyake, C., Tomizawa, K., Endo, T., Tasaka, M., et al. (2004). Cyclic electron flow around photosystem I is essential for photosynthesis. Nature 429, 579-582. doi: 10.1038/nature02598

Murthy, N. M., Ollagnier-de-Choudens, S., Sanakis, Y., Abdel-Ghany, S. E., Rousset, C., Ye, H., et al. (2007). Characterization of Arabidopsis thaliana SufE2 and SufE3: functions in chloroplast iron-sulfur cluster assembly and Nad synthesis. J. Biol. Chem. 282, 18254-18264. doi: 10.1074/jbc.M701428200

Muto, S., and Miyachi, S. (1977). Properties of a protein activator of NAD kinase from plants. Plant Physiol. 59, 55-60. doi: 10.1104/pp.59.1.55

Nikkanen, L., and Rintamaki, E. (2019). Chloroplast thioredoxin systems dynamically regulate photosynthesis in plants. Biochem. J. 476, 1159-1172. doi: 10.1042/BCJ20180707

Noctor, G., Queval, G., and Gakiere, B. (2006). NAD(P) synthesis and pyridine nucleotide cycling in plants and their potential importance in stress conditions. J. Exp. Bot. 57, 1603-1620. doi: 10.1093/jxb/erj202

Okegawa, Y., Kagawa, Y., Kobayashi, Y., and Shikanai, T. (2008). Characterization of factors affecting the activity of photosystem I cyclic electron transport in chloroplasts. Plant Cell Physiol. 49, 825-834. doi: 10.1093/pcp/pcn055

Ort, D. R., and Yocum, C. F. (1996). Oxygenic photosynthesis: The light reactions. Netherlands: Springer.

Palmieri, F., Rieder, B., Ventrella, A., Blanco, E., Do, P. T., Nunes-Nesi, A., et al. (2009). Molecular identification and functional characterization of Arabidopsis thaliana mitochondrial and chloroplastic $\mathrm{NAD}^{+}$carrier proteins. J. Biol. Chem. 284, 31249-31259. doi: 10.1074/jbc.M109.041830

Palmieri, L., Santoro, A., Carrari, F., Blanco, E., Nunes-Nesi, A., Arrigoni, R., et al. (2008). Identification and characterization of ADNT1, a novel mitochondrial adenine nucleotide transporter from Arabidopsis. Plant Physiol. 148, 1797-1808. doi: 10.1104/pp.108.130310

Perez-Ruiz, J. M., Naranjo, B., Ojeda, V., Guinea, M., and Cejudo, F. J. (2017). NTRC-dependent redox balance of 2-Cys peroxiredoxins is needed for optimal function of the photosynthetic apparatus. Proc. Natl. Acad. Sci. USA 114, 12069-12074. doi: 10.1073/pnas.1706003114

Perez-Ruiz, J. M., Spinola, M. C., Kirchsteiger, K., Moreno, J., Sahrawy, M., and Cejudo, F. J. (2006). Rice NTRC is a high-efficiency redox system for chloroplast protection against oxidative damage. Plant Cell 18, 2356-2368. doi: $10.1105 /$ tpc.106.041541

Petriacq, P., de Bont, L., Hager, J., Didierlaurent, L., Mauve, C., Guerard, F., et al. (2012). Inducible NAD overproduction in Arabidopsis alters metabolic pools and gene expression correlated with increased salicylate content and resistance to Pst-AvrRpm1. Plant J. 70, 650-665. doi: 10.1111/ j.1365-313X.2012.04920.x

Pinnola, A., and Bassi, R. (2018). Molecular mechanisms involved in plant photoprotection. Biochem. Soc. Trans. 46, 467-482. doi: 10.1042/BST20170307
Pollak, N., Dolle, C., and Ziegler, M. (2007). The power to reduce: pyridine nucleotides - small molecules with a multitude of functions. Biochem. J. 402, 205-218. doi: 10.1042/BJ20061638

Schippers, J. H., Nunes-Nesi, A., Apetrei, R., Hille, J., Fernie, A. R., and Dijkwel, P. P. (2008). The Arabidopsis onset of leaf death5 mutation of quinolinate synthase affects nicotinamide adenine dinucleotide biosynthesis and causes early ageing. Plant Cell 20, 2909-2925. doi: 10.1105/tpc.107.056341

Selinski, J., and Scheibe, R. (2019). Malate valves: old shuttles with new perspectives. Plant Biol. 21(Suppl. 1), 21-30. doi: 10.1111/plb.12869

Takahashi, S., and Badger, M. R. (2011). Photoprotection in plants: a new light on photosystem II damage. Trends Plant Sci. 16, 53-60. doi: 10.1016/j. tplants.2010.10.001

Takahashi, H., Takahara, K., Hashida, S. N., Hirabayashi, T., Fujimori, T. Kawai-Yamada, M., et al. (2009). Pleiotropic modulation of carbon and nitrogen metabolism in Arabidopsis plants overexpressing the NAD kinase2 gene. Plant Physiol. 151, 100-113. doi: 10.1104/pp.109.140665

Takahashi, H., Watanabe, A., Tanaka, A., Hashida, S. N., Kawai-Yamada, M., Sonoike, K., et al. (2006). Chloroplast NAD kinase is essential for energy transduction through the xanthophyll cycle in photosynthesis. Plant Cell Physiol. 47, 1678-1682. doi: 10.1093/pcp/pcl029

Thormahlen, I., Zupok, A., Rescher, J., Leger, J., Weissenberger, S., Groysman, J., et al. (2017). Thioredoxins play a crucial role in dynamic acclimation of photosynthesis in fluctuating light. Mol. Plant 10, 168-182. doi: 10.1016/j. molp.2016.11.012

Todisco, S., Agrimi, G., Castegna, A., and Palmieri, F. (2006). Identification of the mitochondrial NAD+ transporter in Saccharomyces cerevisiae. J. Biol. Chem. 281, 1524-1531. doi: 10.1074/jbc.M510425200

Turner, W. L., Waller, J. C., Vanderbeld, B., and Snedden, W. A. (2004). Cloning and characterization of two NAD kinases from Arabidopsis. Identification of a calmodulin binding isoform. Plant Physiol. 135, 1243-1255. doi: 10.1104/ pp.104.040428

Van Hoewyk, D., Abdel-Ghany, S. E., Cohu, C. M., Herbert, S. K., Kugrens, P., Pilon, M., et al. (2007). Chloroplast iron-sulfur cluster protein maturation requires the essential cysteine desulfurase CpNifS. Proc. Natl. Acad. Sci. 104, 5686-5691. doi: 10.1073/pnas.0700774104

Vialet-Chabrand, S., Matthews, J. S., Simkin, A. J., Raines, C. A., and Lawson, T. (2017). Importance of fluctuations in light on plant photosynthetic acclimation. Plant Physiol. 173, 2163-2179. doi: 10.1104/pp.16.01767

Waller, J. C., Dhanoa, P. K., Schumann, U., Mullen, R. T., and Snedden, W. A. (2009). Subcellular and tissue localization of NAD kinases from Arabidopsis: compartmentalization of de novo NADP biosynthesis. Planta. 231, 305-317. doi: 10.1007/s00425-009-1047-7

Wedel, N., Soll, J., and Paap, B. K. (1997). CP12 provides a new mode of light regulation of Calvin cycle activity in higher plants. Proc. Natl. Acad. Sci. USA 94, 10479-10484.

Yamori, W., Makino, A., and Shikanai, T. (2016). A physiological role of cyclic electron transport around photosystem I in sustaining photosynthesis under fluctuating light in rice. Sci. Rep. 6:20147. doi: 10.1038/srep20147

Yoshimura, K., and Shigeoka, S. (2014). Versatile physiological functions of the Nudix hydrolase family in Arabidopsis. Biosci. Biotechnol. Biochem. 79, 354-366. doi: 10.1080/09168451.2014.987207

Conflict of Interest Statement: The authors declare that the research was conducted in the absence of any commercial or financial relationships that could be construed as a potential conflict of interest.

Copyright (c) 2019 Hashida and Kawai-Yamada. This is an open-access article distributed under the terms of the Creative Commons Attribution License (CC BY). The use, distribution or reproduction in other forums is permitted, provided the original author(s) and the copyright owner(s) are credited and that the original publication in this journal is cited, in accordance with accepted academic practice. No use, distribution or reproduction is permitted which does not comply with these terms. 